\title{
Damage Identification of HAWT Blade using Ordinary Linear Kriging Method and Variation of Blade's Modal Parameters
}

\author{
Ameen El-Sinawi*, Mohammed Awadallah, Isam Janajreh \\ Mechanical Engineering Department, Khalifa University of Science and Technology, Masdar Institute, PO Box 54224, \\ Masdar City, Abu Dhabi, United Arab Emirates
}

\begin{abstract}
Wind turbine blades operate in a harsh environment causing them to always be susceptible to damage. Variable wind loading, debris impact, and thermal gradient, among other factors, can cause damage to the blades. Detection of blade damage at early stages can prevent massive cost associated with turbine down-time and blade replacement. In this work, a vibration-based method is presented to detect damage at early stages. The presented method takes advantage of the effect of crack on modal parameters of the blades vibration. Finite element model (FEA) is constructed for both healthy and damage blade to study that effect. Power spectral density (PSD) plots of the blade's vibration before and after damage are compared and the changes in the resonant modal amplitudes frequencies are identified. To minimize the number accelerometers needed to monitor the health of the blade and without compromising the accuracy of damage predictions, ordinary kriging method is used to predict cracks in the blade's structure. Kriging uses modal parameter data, experimental or otherwise, to estimate damage location on the blade. It creates a map of damage predictions throughout the region use measurements from far less sensors than common techniques. Damage characteristics estimates using the proposed method showed damage attributes predictions with accuracy greater than $93 \%$. Simulation is used to validate the proposed method and the results are discussed.
\end{abstract}

Keywords: Vibration Analysis, Wind Turbine Damage, Kriging Analysis, Model Parameters, FEA, BEMM method

\section{Introduction}

Wind energy is the main source of renewable energy with annual growth of nearly $20 \%$ in the past two decades $[1,2]$. However, as it is the case with all other energy conversion methods, wind energy harvesting has its challenges. The latter are mainly due to lack of historical wind data or premature failure of wind turbine tied to rotational unbalance, erosion, corrosion and impact failure, and its earlier detection and remedy $[1,2]$.

Vibration analysis can be used as a potential technique for damage identification of wind turbine blades through the monitoring of variation of resonant frequencies and mode shapes of the blade structure. Damage inflected on the blade directly affects its vibrational behavior and manifest itself in the form of changes in resonant frequencies and mode shapes. Vibration-

${ }^{*}$ Corresponding author. Tel.: +97126075509

E-mail: ameen.elsinawi@ku.ac.ae

(C) 2018 International Association for Sharing Knowledge and Sustainability

DOI: $10.5383 /$ ijtee.17.01.007 based methods use accelerometers to monitor the vibration of the structure. The number of accelerometers attached to the structure depends on the detection approach, the size of the structure and the environment in which the structure operates. A large body of research work in this field focuses on damage identification using vibration signals from the structure.

The fact that large wind turbines are located in remote locations such as off shore or uphill, where their access is limited, remote detection of their onset of "mal-performance" is of great importance. In general, turbines may underperform due to uncompromised blade/rotor geometrical change or faulty mechanical or electrical systems. The latter two types are easy identified by comparison with operational turbine. However, early detection of the former enables swift maintenance and ensures steady operation. 
Wind turbines are subjected to fluctuating environmental conditions that may also initiate and cause damages to the blades, rotor, or hidden rotating components [3]. Blade and rotor damages imply high economical cost in service and down-time. Therefore, mitigation of such failures through simulation and modeling as well as early online detection via remote sensing reduces the risk of wind turbine failure, cuts cost and increases their deployments as an efficient renewable and clean source of energy.

Numerous researchers implemented damage detection techniques using radar [4], fiber optics [5, 6], laser [7, 8], acoustic $[9,10]$ and thermography and imaging [11-13] for damage detection. Others used Kriging analysis as it revealed higher accuracy. Padopoulos et al [14] and Garcia et al [15] implemented the microscopic length deformation at pre-selected points in the structure to infer information about the total deformation of the whole structure. As the total deformation depends on the magnitude of damages and their location, this technique introduces non-isotropy to the structure and poses strong drawback.

Ultrasonic Testing (UT) has been used for wind turbine blades damage detection. Raisutis et al [13] used guided waves ultrasonic air-coupled technique, to detect defects in a structure and record its acoustic response. Wavelet transform of the received waveform was used to monitor and analyze any internal defects. The inspected region size, however, is limited and localized and failure location can be occurred elsewhere.

Because small changes in the structure or support will cause changes in the vibration signature, the PSD techniques demonstrate its uniqueness in addition to its relatively low cost [16]. Furthermore, vibration-based structural monitoring methods give instantaneous feedback on the condition of blades [17]. Monitoring natural frequencies and mode shapes is a very basic tool for detecting faults. It can be achieved by accessing only few points in the structure and is exposed to less experimental noise [18]. Shi et al [19] experimentally examined the ability of natural frequencies and mode shape changes in detecting fine cracks. Their results revealed that the mode shape change approach can detect cracks up to ten times finer than frequency shifts approach. They suggested that identifying defects relying on natural frequencies shifts is inconclusive. Also, applying mode shapes approach only, requires many sensors distributed along the structure [20]. Therefore, utilizing both natural frequencies and mode shape changes would complement one the other.

Drawbacks of previous methods can be attributed to the use of expensive equipment such as radar and laser, noisy environment and lack of accuracy in the case of acoustic and thermography, reliability of the measurement devices as it is the case with brittle fiber optics and localized and tedious inspection methods such as the UT.

In this work, a dynamic model is constructed for both, healthy and defected turbine blades by introducing a crack into the test turbine's blade. The aerodynamic load on the wind turbine is evaluated based on the Blade Element Momentum-integral Method (BEMM). According to the BEMM the blade's radial direction is subdivided into multiple elements where the drag and lift forces are evaluated and are used as forces input to the dynamic turbine model. The Power Spectral Density (PSD) is monitored corresponding to every defect dimensions and location to draw a relation between crack parameters and PSD changes. Furthermore, Kriging analysis is used to assess the intensity of these damages and relate the output of the PSD to the introduced crack parameters.

\section{Structural Modeling and Aerodynamic Load}

\subsection{Structural Modeling}

Fig. 1 shows the FEA model of blade-hub assembly generated by ANSYS. The blades are of NACA-4415 type airfoil and their discretized mesh consists of 36,374 tetrahedron elements and 70,417 nodes (n). The lower surface of the hub shown in the Fig. 1 is constrained/fixed in all directions. Knowing that $\mathrm{n}$ is usually large, a nodal model implies huge numerical burden, making it impractical to produce a working state-space model suitable for structural simulation, estimation and control applications. Thus, $\mathrm{N}$ dimensional second-order modal model can be used instead, where $\mathrm{N}<<\mathrm{n}$ is number of modes included in the modal dynamic model [21]. This can be described per Eq. 1 and Eq. 2 as:

$$
\begin{aligned}
& \ddot{\boldsymbol{\eta}}++\mathbf{2 Z} \boldsymbol{\Omega} \dot{\boldsymbol{\eta}}+\boldsymbol{\Omega}^{2} \boldsymbol{\eta}=\boldsymbol{B}_{\boldsymbol{m}} \boldsymbol{u} \\
& Y_{s}=C_{m d} \eta+C_{m v} \dot{\boldsymbol{\eta}}
\end{aligned}
$$

where $\boldsymbol{\eta}=\boldsymbol{\phi} \boldsymbol{q}, \boldsymbol{\phi}$ is the $\mathrm{n} \times \mathrm{N}$ modal matrix, $\Omega$ is the $\mathrm{N} \times \mathrm{N}$ diagonal matrix of Modal frequencies, $\mathrm{Z}$ is the $\mathrm{N} \times \mathrm{N}$ modal damping matrix, $\mathrm{B}_{\mathrm{m}}$ is the $\mathrm{N} \times \mathrm{p}$ modal input matrix, $\boldsymbol{C}_{\boldsymbol{m} \boldsymbol{d}}$ is $1 \times \mathrm{N}$ modal displacement matrix, and $\boldsymbol{C}_{\boldsymbol{m} v}$ is $1 \times \mathrm{N}$ modal velocity matrix, where $p$ and 1 are the number of inputs and outputs, respectively. Thus, the linear, time-invariant (LTI) modal model is:

$$
\begin{aligned}
& \dot{\boldsymbol{z}}=\boldsymbol{A z}+\boldsymbol{B} \\
& Y_{s}=C z+G u
\end{aligned}
$$

The new state vector $\underline{Z}=\left[\underline{\boldsymbol{Z}}_{1}, \underline{\mathbf{z}}_{2}\right]^{T}=[\boldsymbol{\eta} \boldsymbol{\eta}]^{T}$ is introduced such that, the state-space representation of the structure having point forces as inputs and point accelerations as outputs. Matrices of Eqs. (3-4) are, $\boldsymbol{A}=\left[\begin{array}{cc}\mathbf{0} & \mathbf{1} \\ -\mathbf{\Omega}^{2} & -\mathbf{2 Z \Omega}\end{array}\right], \boldsymbol{B}=\left[\begin{array}{ll}\mathbf{0} & \boldsymbol{B}_{m}\end{array}\right]^{T}, \boldsymbol{C}=$ $\left[\begin{array}{ll}-C_{m a} \Omega^{2} & -2 C_{m a} Z \Omega\end{array}\right], G=C_{m a} B_{m}, C_{m a}=C_{a a} \Phi$ and $C_{a a}$ is the accelerometer locations matrix. Assuming proportional damping, then $Z$ and $\Omega$ are the matrices of modal damping and modal frequencies. Model defined in Eqs. (3-4) represent the full-order modal model of the structure. This is usually a large model if the structure is large, and if large number of modes is incorporated into the model. Therefore, the numerical cost associated with implementing such model for vibration estimation and vibration analysis is still high, although it is considerably less than the nodal model of Eqs. (1-2). Hence, model reduction is an option, provided that the model accuracy is maintained. The dynamic model of the hub-blade structure is excited by the aerodynamic load presented in the following section. 


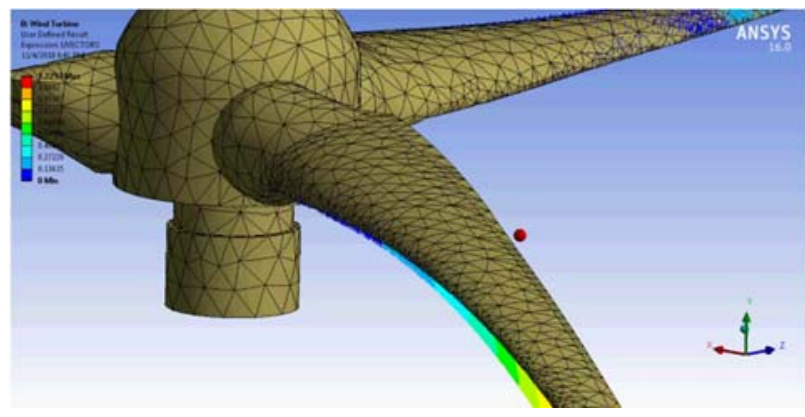

Fig. 1. FE model and mesh of the blade-hub assembly, the blades are NACA-4415 type airfoil, and the hub is constrained in all directions at the lower surface

\subsection{Aerodynamic Calculation}

Blade Element Momentum-integral Method (BEMM) is a simplified flow field tool for evaluation of the aerodynamic load from the lift \& drag coefficients of a given turbine airfoil [22]. The evaluated load is applied at multiple sections $(L \approx 10)$ along the span of the turbine blade. The angle of attack accounts for the incoming wind and rotor speeds. Higher fidelity methods such as Navier-Stokes flow simulation or wind tunnel experimental data on rotating turbine can also substitute full scale data [23]. Processing this data enables the application of a reasonable multiple fixed-point load along the blade span for further model analysis. Specifically, the forces on the wind turbine blades is expressed as function of the coefficients of lift $\left(C_{L}\right)$ and drag $\left(C_{d}\right)$ as per the angle shown in Fig. 2. The blade is assumed to be discretized into $L$-sections; each has its own force, all of which are collectively added to obtain the individual blade's total force. As theory assumes no aerodynamic interaction between sections, the radial flow is ignored. Moreover, forces on the blades are determined solely by the airfoil shape. The increments of the thrust $\left(d F_{T}\right)$ and the torque $\left(d F_{N}\right)$ are evaluated per Eq. 5 and 6 as:

$$
\begin{aligned}
& d F_{T}=d F_{L} \cos \varphi+d F_{D} \sin \varphi \\
& d F_{N}=d F_{L} \sin \varphi-d F_{D} \cos \varphi
\end{aligned}
$$

where $d F_{L}=C_{l} \frac{1}{2} \rho U_{r e l}^{2} c d r, d F_{D}=C_{d} \frac{1}{2} \rho U_{r e l}^{2} c d r, \varphi$ is the angle of relative wind, $U_{r e l}$ is the relative wind speed, $C_{l}$ and $C_{d}$ are the lift and drag coefficients, respectively. This lead to the expression of the incremental thrust and torque as:

$$
d T=\sigma^{\prime} \pi \rho \frac{U^{2}(1-a)^{2}}{\sin ^{2} \varphi}\left(C_{l} \cos \varphi+C_{d} \sin \varphi\right) r d r
$$

$d N=\sigma^{\prime} \pi \rho \frac{U^{2}(1-a)^{2}}{\sin ^{2} \varphi}\left(C_{l} \sin \varphi-C_{d} \cos \varphi\right) r^{2} d r$

where $\sigma^{\prime}$ is the local solidity defined as $\sigma^{\prime}=\mathrm{Bc} / 2 \pi \mathrm{r}$; Here $B$ is the number of blades and $c$ is the blade chord length, $\varphi$ is the angle of relative wind. The shaft torque $\left(N^{*}\right)$ is the sum of $\mathrm{N}_{\mathrm{i}}$ for all the blade sections, and the wind turbine power is the product of $N^{*}$ and $\omega$. Eventually, the wind turbine power prediction lies in solving the axial and rotational induction factors $a$ and $a^{\prime}$ [24], and after some manipulation these are related and given as:

$$
a=\frac{1}{1+\frac{4 \sin ^{2} \varphi}{\sigma^{\prime}\left(C_{l} \cos \varphi+C_{d} \sin \varphi\right)}}, a^{\prime}=a \frac{C_{l} \sin \varphi-C_{d} \cos \varphi}{\left(C_{l} \cos \varphi+C_{d} \sin \varphi\right) \lambda_{s}}
$$

where $\lambda_{s}$ is the local tip speed ratio defined as $\lambda_{s}=\omega r / U$. Solving for the induction factors enables back substitution and evaluation of the forces exerted on the rotor and prediction the turbine performance.

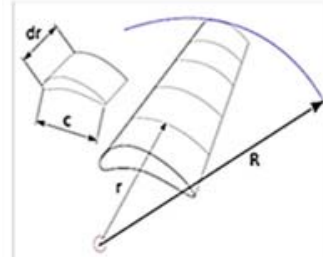

(a)

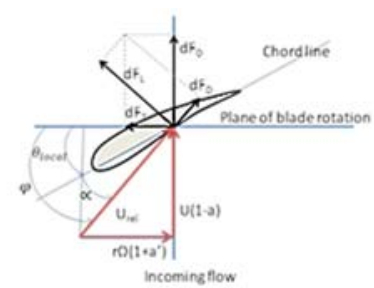

(b)
Fig. 2. Force direction on the blades cross-section, (a) shapes of the sections on which forces are calculated (b) forces generated from wind loading and their direction on each cross-section

\section{Kriging Analysis}

\subsection{Ordinary Kriging}

Kriging is a method that allows the estimation of the value of variable at certain grid points within a region using linear combination of available measurement of the variable at neighboring points. The estimates are a weighted sum of known values of the variable. Kriging method uses the covariancedistance data in the interpolation to determine the weights in the sum. Kriging is considered to be the best linear unbiased estimator. It is unbiased because the estimation error average is zero while it is the best estimator because estimates error variance is minimized. Moreover, kriging requires that the analyzed data must be stationary [25].

\subsection{Experimental Variogram}

The semivariogram is a measure of the degree of spatial dependence between samples. It is usually a function of the form given in Eq. 1 where the spatial correlation of a set of random variables as a function of the separation distance between those data points is expressed in the following form;

$\gamma(d)=\frac{1}{2 n(d)} \sum_{i=1}^{n}\left[Z\left(x_{i}\right)-Z\left(x_{i}+d\right)\right]^{2}$

where $\gamma(d)$ is the experimental semivariogram, $d$ is the separation distance, $Z\left(x_{i}\right)$ is the value of the observation at location $x_{i}, Z\left(x_{i}+d\right)$ is the value of the observation at location $x_{i}+d$, and $n(d)$ is the number of distinct pairs separated by distance $d$. A semivariogram might be thought of as "dissimilarity between point values as a function of distance", such that the dissimilarity is greater for points that are farther apart. Therefore, after a certain distance between points the correlation between points becomes zero. The latter is called the range $(R)$. The value at which the correlation between points is zero is called the sill $(s)$, where the variance of the studied parameter reaches its maximum value. The value of $\gamma(d=0)$ is called the nugget $(N)$ where the error is only caused by measurement. 


\subsection{Theoretical Semivariogram}

The experimental semivariogram usually does not have the isotropy property (i.e., uniformity in all directions). Therefore, semivariogram fitted to experimental data can be used instead. The fitted model can be linear, gaussian, spherical, or exponential, where in all of them the values of the nugget $(N)$, sill $(S)$, and range $(R)$, are determined. The choice of fitted model depends on the nature of observation data and the performance and accuracy of the estimates generated by each model. IN this work, ordinary linear Kriging model is chosen since the simulation data is usually accurate and have no noise contamination. Thus, $\hat{\gamma}_{i j}(d)$ given in Eq. (11);

$\hat{\gamma}_{i j}(d)=\left\{\begin{array}{c}N+S\left(\frac{d}{R}\right), 0<d \leq R \\ N+S, \quad d>R\end{array}\right.$

The kriging equation can be expressed as the summation shown in Eq. 3, such that;

$$
\hat{\gamma}_{i o}=\sum_{j=1}^{n} \omega_{i} \hat{\gamma}_{i j}+\lambda
$$

where $\hat{\gamma}_{i o}$ are the variogram values given by Eq. 2 between all known points $\left(Z_{i}\right)$ and unknown point $\left(Z_{o}\right), \omega_{i}^{\prime} s$ are found by solving the system of linear equations shown in Eq. 4;

$$
\left[\begin{array}{cccc}
\hat{\gamma}\left(Z_{1}-Z_{1}\right) & \ldots & \hat{\gamma}\left(Z_{1}-Z_{n}\right) & 1 \\
\vdots & \ddots & \vdots & \vdots \\
\hat{\gamma}\left(Z_{n}-Z_{1}\right) & \ldots & \hat{\gamma}\left(Z_{n}-Z_{n}\right) & 1 \\
1 & \ldots & 1 & 0
\end{array}\right] \cdot\left[\begin{array}{c}
\omega_{1} \\
\vdots \\
\omega_{n} \\
\lambda
\end{array}\right]=\left[\begin{array}{c}
\hat{\gamma}\left(Z_{o}-Z_{1}\right) \\
\vdots \\
\hat{\gamma}\left(Z_{o}-Z_{n}\right) \\
1
\end{array}\right]
$$

Lagrange multiplier $\lambda$ plays a key role in minimizing the estimation error (i.e. minimizing the kriging variance). To generate unbiased estimates of the variable at unknown points, the total sum of weights must add up to 1 as shown in Eq.11.

$$
\sum_{i=1}^{n} \omega_{i}=1
$$

Kriging yields an estimation of the variable $Z$ at a given point as well as the estimation error variance, where the latter gives a good indicator of the relative quality of estimations. The error variance $\sigma_{e}^{2}$ is, [25].

$\sigma_{e}^{2}=\sum_{i=1}^{n} \omega_{i} \hat{\gamma}_{i}+\lambda$

Depending on the size, location and orientation of the turbine blade's structural damage, certain modes of vibration are affected. Therefore, we have to know first how many mode shapes are necessary to obtain valuable and sufficient information about mode shapes and natural frequencies affected by the range of the dimensions and locations of the crack. Through data analysis technique used in this research, it is possible to locate and identify any crack using the vibration signature of wind turbine when excitation forces are applied.

\begin{tabular}{|c|c|c|c|c|}
\hline \multirow{2}{*}{ Force } & \multicolumn{4}{|c|}{ Wind Speed } \\
\hline & $5 \mathrm{~m} / \mathrm{s}$ & $8 \mathrm{~m} / \mathrm{s}$ & $10 \mathrm{~m} / \mathrm{s}$ & $12 \mathrm{~m} / \mathrm{s}$ \\
\hline$d F_{N} 1$ & 0.240 & 0.386 & 0.506 & 0.645 \\
\hline$d F_{T} 1$ & 0.594 & 1.338 & 2.000 & 2.803 \\
\hline$d F_{N} 2$ & 0.339 & 0.495 & 0.612 & 0.743 \\
\hline$d F_{T} 2$ & 0.653 & 1.439 & 2.129 & 2.961 \\
\hline$d F_{N} 3$ & 0.420 & 0.593 & 0.717 & 0.851 \\
\hline$d F_{T} 3$ & 0.621 & 1.373 & 2.029 & 2.817 \\
\hline$d F_{N} 4$ & 0.465 & 0.646 & 0.773 & 0.908 \\
\hline$d F_{T} 4$ & 0.554 & 1.222 & 1.806 & 2.508 \\
\hline$d F_{N} 5$ & 0.507 & 0.687 & 0.815 & 0.950 \\
\hline$d F_{T} 5$ & 0.499 & 1.085 & 1.597 & 2.214 \\
\hline$d F_{N} 6$ & 0.554 & 0.728 & 0.855 & 0.988 \\
\hline$d F_{T} 6$ & 0.463 & 0.980 & 1.433 & 1.977 \\
\hline$d F_{N} 7$ & 0.613 & 0.780 & 0.905 & 1.038 \\
\hline$d F_{T} 7$ & 0.440 & 0.904 & 1.308 & 1.793 \\
\hline$d F_{N} 8$ & 0.666 & 0.822 & 0.942 & 1.070 \\
\hline$d F_{T} 8$ & 0.423 & 0.844 & 1.208 & 1.643 \\
\hline$d F_{N} 9$ & 0.722 & 0.867 & 0.981 & 1.105 \\
\hline$d F_{T} 9$ & 0.410 & 0.797 & 1.128 & 1.523 \\
\hline
\end{tabular}

Table 2. Aerodynamic forces calculated by Eqs, (5) and (6) on each blade section for each wind velocity

This is accomplished by the constructed dynamic model that based on the Eigen values and Eigen vectors obtained from high fidelity finite element (FE) model of the turbine blade for each of the pristine/healthy and defected turbine blades. State space models constructed from Eigen values and Eigen vectors are used to generate the PSD of the blade under various forcing and defect conditions. Turbine blade tested in this study has a length of $1.22 \mathrm{~m}$ made by ABSPLUS-P430 Inc. and characterized with ultimate, yield, and modulus tensile stresses of $33 \mathrm{MPa}, 32 \mathrm{MPa}$, and $2.2 \mathrm{GPa}$, respectively. Hub and blade dimensions and

\begin{tabular}{|c|c|c|}
\hline Component & Element & Dimension [mm] \\
\hline \multirow[t]{6}{*}{ Hub } & Hub & 300.00 \\
\hline & Diameter & \\
\hline & Hub & 10.00 \\
\hline & Thickness & \\
\hline & Shaft & 146.60 \\
\hline & Diameter & \\
\hline \multirow[t]{2}{*}{ Blade } & Total & 1220.00 \\
\hline & length & \\
\hline \multirow[t]{2}{*}{ 3-balded } & Maximum & 176.30 \\
\hline & Width & \\
\hline \multirow[t]{2}{*}{ Airfoil } & Tip Width & 75.62 \\
\hline & NACA-4415 & ----- \\
\hline
\end{tabular}
configurations are summarized in Table 1.

Table 1. Mechanical properties of the Hub-Blade assembly 


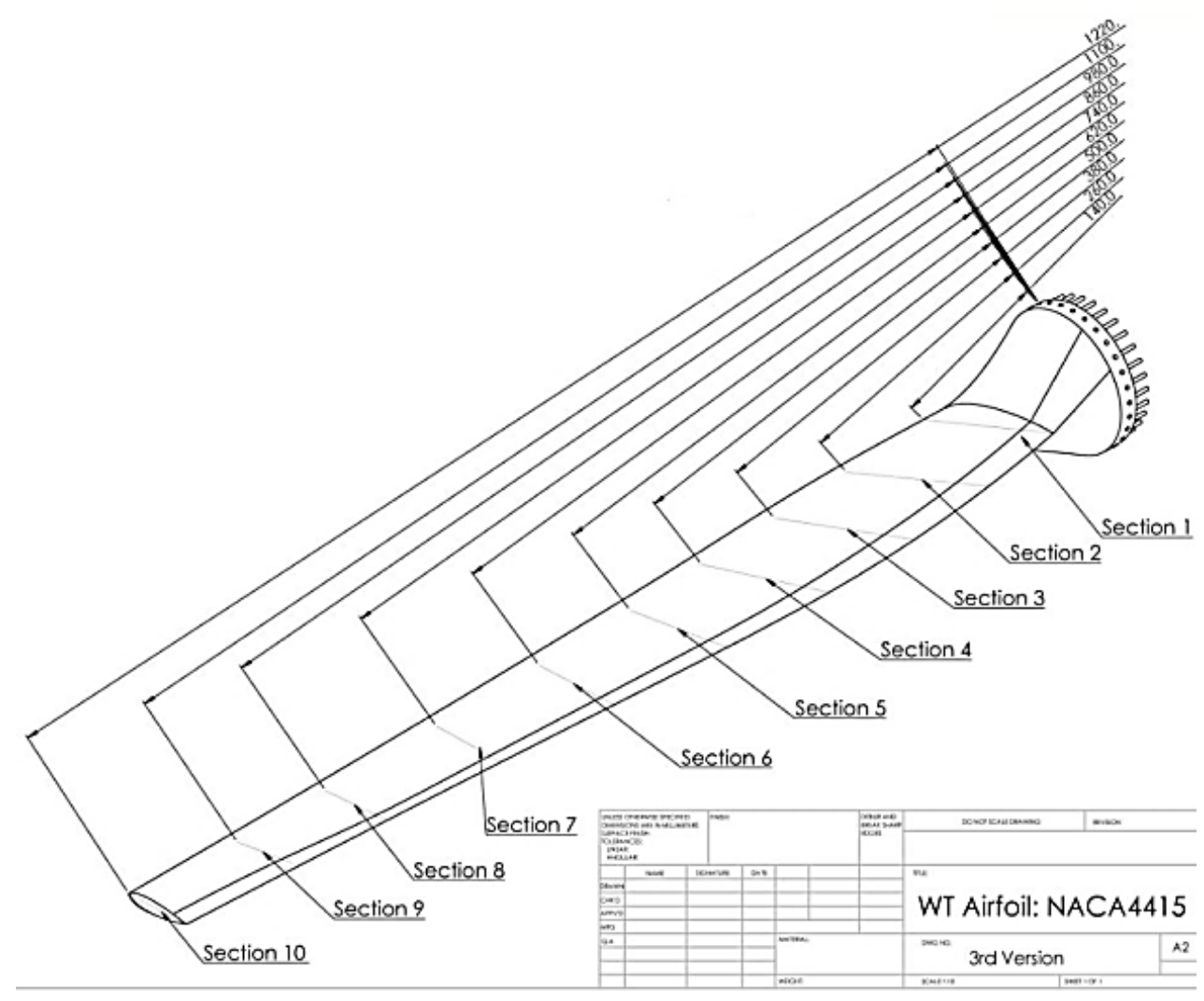

Fig. 3. Wind turbine's blade drawing with all sections

\section{Simulation Results I}

\subsection{Aerodynamic forces}

In simulation, the wind turbine is subjected to four different wind speeds namely $5,8,10$ and $12 \mathrm{~m} / \mathrm{s}$. The wind turbine's blade is divided into 9 sections of equal length $(0.12 \mathrm{~m})$ as shown in Fig. 3. Following the BE-LMM procedures, the aerodynamic forces are evaluated (FT, FN) as per Table 2.

\subsection{Simulation test procedure}

Blade models are built for eight different crack dimensions and locations in accordance with experimental matrix given in Table 4. The crack identity depends on three factors, i.e. Crack Length (L), Crack Width (W), and Crack Location (C). In order to make the results useful for most of blade turbines sizes, a $2^{k}$ factorial experiment is designed with each factor represented as a percentage of the blade length, and has two levels, namely, higher level (+) and lower level (-). In this model, the higher and lower values of the crack length (L), width (W), and location (C) are given in Table 3 . Table 4 summarizes the level combinations of the $2^{k}$ factorial experiment.

Table 3. Crack length, width and locations extreme values

\begin{tabular}{lll}
\hline Factor & Higher level ( + ) [mm] & Lower level ( - ) [mm] \\
\hline Length (L) & 15.00 & 10.00 \\
Width (W) & 1.00 & 0.50 \\
$\begin{array}{l}\text { Location } \\
\text { (C) }\end{array}$ & 1000.00 & 500.00 \\
\hline
\end{tabular}

Table 4. $2^{k}$ factorial experiment crack dimension and locations

\begin{tabular}{ccccc}
\multicolumn{5}{c}{ matrix } \\
\hline Blade ID & L & W & C & Total \\
\hline Healthy & N/A & N/A & N/A & N/A \\
$\mathbf{1}$ & + & + & + & +++ \\
$\mathbf{2}$ & - & + & + & -++ \\
$\mathbf{3}$ & + & - & + & +-+ \\
$\mathbf{4}$ & - & - & + & --+ \\
$\mathbf{5}$ & + & + & - & ++- \\
$\mathbf{6}$ & - & + & - & -+- \\
$\mathbf{7}$ & + & - & - & +-- \\
$\mathbf{8}$ & - & - & - &.- \\
\hline
\end{tabular}

\section{Simulation Results II}

The PSD is produced for three fixed accelerometer measurements, each of which is located at a specific point on the blade's surface at (a) Hub center, (b) blade middle span and (c) blade tip as shown in Fig.4. State-space models for the eight cases listed in Table 4 are constructed from Eigen values and Eigen vectors generated by ANSYS Finite element method (FEM). The simulation shows that acceleration response in the direction perpendicular to the blades surface (i.e., Y-direction) is the most affected by the induced damages compared to the 
radial direction. The radial direction has negligible changes in amplitudes and frequencies before and after damage is introduced to the blade. Figs. 5 and 6 show sample PSD's of the accelerometers at the middle and tip, respectively. Changes in modal amplitudes and frequencies are present when damage is introduced to the blade. Latter figures also indicate that damage effect on amplitudes is much more pronounced than that on the frequencies. However, both changes in resonant amplitudes and frequencies will be utilized in the Kriging method to predict damage attributes at points other than sensor locations. The results suggest that the values of each crack parameters, influences the PSD amplitudes, and to a lesser degree, frequencies.

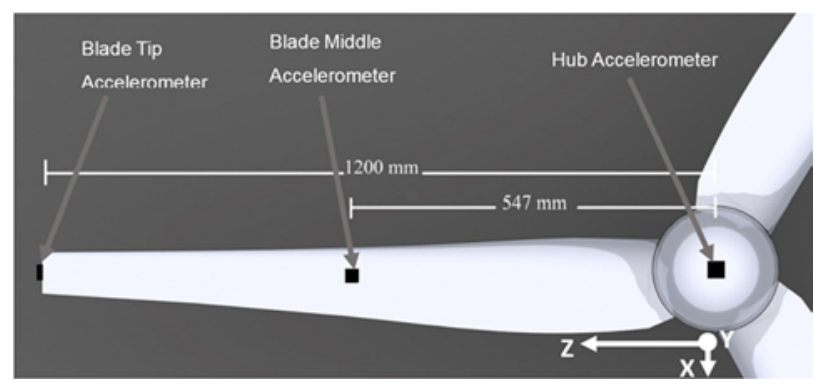

Fig. 4. Sensor locations on the surface of the blade, global directions are also shown in the figure and the acceleration is measured in the Y-direction

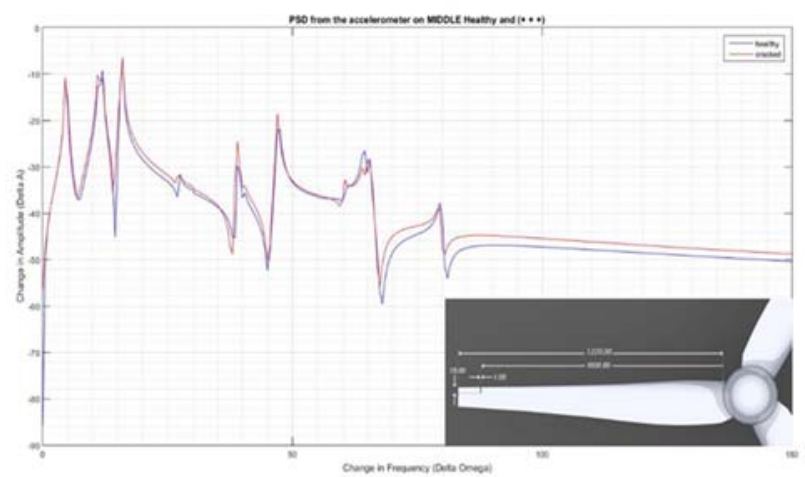

Fig. 5. Power Spectral Density of simulated blade with crack levels $(+++)$ obtained from the sensor at the middle of the blade

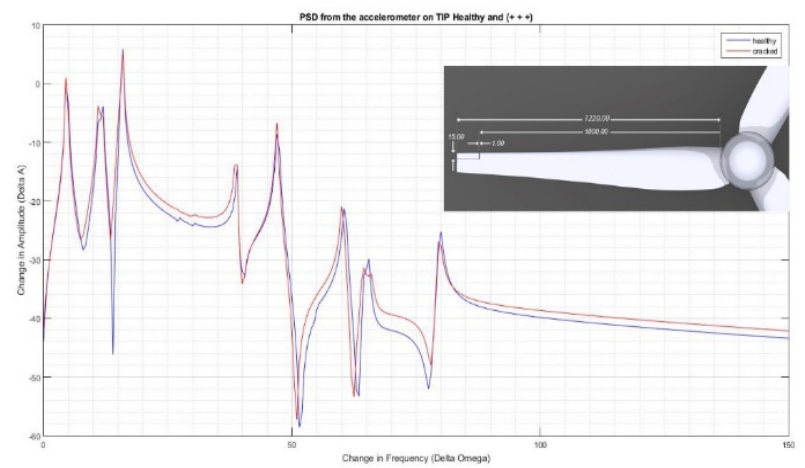

Fig. 6. Power Spectral Density of simulated blade with crack levels $(+++)$ obtained from the sensor at the tip of the blade
The Kriging analysis prediction and their comparison with the estimated length, width and location to the induced cracks are summarized in table 5. For the sake of saving space, Table 5 only lists the Kriging results for two of the given wind speeds however, the other two have also produced similar results. The validation is done using a simulated crack length of $11 \mathrm{~mm}$ and width of $0.78 \mathrm{~mm}$ at $720 \mathrm{~mm}$ radial location from hub center. The table summarizes the estimated length, width and location of the damage for two of the given wind speeds, namely 5 and $10 \mathrm{~m} / \mathrm{s}$ and at the three sensors' locations. The error, in some cases, is as low as $0.17 \%$ indicating significant improvement in accuracy compared to existing techniques. Conventional techniques typically produce errors in the range of $5-8 \%$ in detecting crack dimensions and location in wind turbines. It is noticed that higher frequencies revealed more accurate estimation of crack properties, especially crack location. Table 5 shows that at $39 \mathrm{~Hz}$ resonant frequency, the error is around $15.7 \%$ while at $16 \mathrm{~Hz}$ resonant frequency, it is near $17.4 \% .2$. The average error estimated for PSD by Kriging from the response obtained from the TIP sensor is lower than MID and HUB sensors. TIP sensors at higher frequency revealed an average error of $6.5 \%$ while it was $20.0 \%$ for each of the HUB and MID sensors.

Table. 5 also indicates reveals some interesting results that can be utilized in damage identification of wind turbine blades. The results are summarized by the following;

1. At lower wind speeds (i.e. $5 \mathrm{~m} / \mathrm{s}$ ) the tip accelerometer has the best predictions at higher resonant frequencies where the maximum error is only $3.1 \%$ at $39 \mathrm{~Hz}$ frequency

2. At higher wind speeds, the tip accelerometer has the best estimates at lower resonant frequency where the error at $16 \mathrm{~Hz}$ has a maximum value of $4.5 \%$

3. The middle accelerometer had the worst damage estimates for all resonant frequencies at all wind speeds

The inverse relationship between wind speed and resonant frequencies error estimates are interesting and calls for further research and analysis. It appears though as if the middle accelerometer is placed at the nodes of certain mode shapes where the acceleration is low frequency modes to the point where detection of damage is not possible using accelerometer at that location. 
Table 4. Kriging analysis prediction and error estimation

\begin{tabular}{|c|c|c|c|c|c|}
\hline $\begin{array}{l}\text { Wind } \\
\text { Speed }\end{array}$ & $\begin{array}{l}\text { Natural } \\
\text { Freq. }\end{array}$ & $\begin{array}{l}\text { Sensor } \\
\text { location }\end{array}$ & $\begin{array}{l}\text { Damage } \\
\text { attribute }\end{array}$ & $\begin{array}{l}\text { Attribute } \\
\text { Value }\end{array}$ & $\begin{array}{l}\text { Estimation } \\
\text { Error }\end{array}$ \\
\hline \multirow[t]{18}{*}{$5 \mathrm{~m} / \mathrm{s}$} & \multirow[t]{9}{*}{$16 \mathrm{~Hz}$} & \multirow[t]{3}{*}{ HUB } & (L) & 13.2 & $20.4 \%$ \\
\hline & & & (W) & 0.825 & $5.8 \%$ \\
\hline & & & (C) & 612.1 & $15.0 \%$ \\
\hline & & \multirow[t]{3}{*}{ MID } & (L) & 14.0 & $27.4 \%$ \\
\hline & & & (W) & 0.948 & $21.6 \%$ \\
\hline & & & (C) & 875.8 & $21.6 \%$ \\
\hline & & \multirow[t]{3}{*}{ TIP } & (L) & 14.9 & $35.3 \%$ \\
\hline & & & (W) & 0.968 & $24.1 \%$ \\
\hline & & & (C) & 988.5 & $37.3 \%$ \\
\hline & \multirow[t]{9}{*}{$39 \mathrm{~Hz}$} & \multirow[t]{3}{*}{ HUB } & (L) & 13.3 & $21.0 \%$ \\
\hline & & & (W) & 0.802 & $2.9 \%$ \\
\hline & & & (C) & 579.4 & $19.5 \%$ \\
\hline & & \multirow[t]{3}{*}{ MID } & (L) & 10.2 & $7.3 \%$ \\
\hline & & & (W) & 0.997 & $27.8 \%$ \\
\hline & & & (C) & 519.3 & $27.9 \%$ \\
\hline & & \multirow[t]{3}{*}{ TIP } & (L) & 11.0 & $2.0 \%$ \\
\hline & & & (W) & 0.764 & $2.0 \%$ \\
\hline & & & (C) & 742.2 & $3.1 \%$ \\
\hline \multirow{18}{*}{$\begin{array}{l}10 \\
\mathrm{~m} / \mathrm{s}\end{array}$} & \multirow[t]{9}{*}{$16 \mathrm{~Hz}$} & \multirow[t]{3}{*}{ HUB } & (L) & 11.3 & $3.0 \%$ \\
\hline & & & (W) & 0.745 & $4.5 \%$ \\
\hline & & & (C) & 698.4 & $3.0 \%$ \\
\hline & & \multirow[t]{3}{*}{ MID } & (L) & 13.9 & $26.2 \%$ \\
\hline & & & (W) & 0.980 & $25.6 \%$ \\
\hline & & & (C) & 810.7 & $12.6 \%$ \\
\hline & & \multirow[t]{3}{*}{ TIP } & (L) & 10.1 & $8.2 \%$ \\
\hline & & & (W) & 0.522 & $33.1 \%$ \\
\hline & & & (C) & 652.5 & $9.4 \%$ \\
\hline & \multirow[t]{9}{*}{$39 \mathrm{~Hz}$} & \multirow[t]{3}{*}{ HUB } & (L) & 10.0 & $9.1 \%$ \\
\hline & & & $(W)$ & 1.000 & $28.2 \%$ \\
\hline & & & (C) & 999.1 & $38.8 \%$ \\
\hline & & \multirow[t]{3}{*}{ MID } & (L) & 11.3 & $2.5 \%$ \\
\hline & & & (W) & 0.879 & $12.7 \%$ \\
\hline & & & (C) & 614.5 & $14.7 \%$ \\
\hline & & \multirow[t]{3}{*}{ TIP } & (L) & 12.3 & $11.7 \%$ \\
\hline & & & (W) & 0.838 & $7.4 \%$ \\
\hline & & & (C) & 784.6 & $9.0 \%$ \\
\hline
\end{tabular}

\section{Conclusion}

Damage identification in wind turbine blades using change of resonant amplitudes and frequencies found from Power Spectral Density was studied. Blade Element Momentum-integral Method is used to generate the aerodynamic blade load and FEM is used to generate the Eigen values and Eigen vectors needed for the construction of the turbine blade's dynamic model. Eight different blade damages or crack characteristics were induced as per the required $\mathbf{2}^{\boldsymbol{K}}$ factorial experiment. Simulation is carried out where the blades are excited by the aerodynamic forces and the acceleration response was generated at three locations along the blade's span. Results suggest that acceleration of the blade is sensitive to damage mostly along the rotor's axis direction. Small cracks (relative to blade's length) have more effect on the blades resonant amplitude than blade resonant frequencies at all modes. Crack length, width and distance from hub center have considerable influence on the PSD amplitudes. Kriging analysis revealed that using Kriging and PSD amplitudes of higher resonant frequencies results in more accurate estimation of crack location. It shows that at low wind speeds, the PSD amplitudes of higher resonant frequencies generated lower error in damage attributes estimation. However, the opposite is found to be true for higher wind speeds where the error estimates were lower at lower resonant frequencies. It was also noticed that the lower wind speeds, the tip accelerometer generated the lowest error while at higher wind speeds, the hub estimates had the lowest errors. The overall error for all wind speeds at $39 \mathrm{~Hz}$ was found $15.7 \%$ while is $17.4 \%$ at $16 \mathrm{~Hz}$. It is clear that kriging analysis requires experimental data or prior knowledge of the relationship between damage attributes and the PSD's to construct the variance and estimation models. This is a drawback in cases where no previous vibration data is available for a specific blade.

\section{Acknowledgments}

Authors acknowledge the support of Khalifa University of Science and Technology (KUST), Abu Dhabi, UAE.

\section{References}

[1] D. S. Li, S. C. M. Ho, G. B. Song, L. Ren, and H. N. Li, "A review of damage detection methods for wind turbine blades," Smart Materials and Structures, vol. 24, Mar 2015.

[2] W. X. Yang, Z. K. Peng, K. X. Wei, and W. Y. Tian, "Structural health monitoring of composite wind turbine blades: challenges, issues and potential solutions," Iet Renewable Power Generation, vol. 11, pp. 411-416, Mar 152017.

[3] J. Ribrant and L. M. Bertling, "Survey of failures in wind power systems with focus on Swedish wind power plants during 1997-2005," Ieee Transactions on Energy Conversion, vol. 22, pp. 167-173, Mar 2007.

[4] J. Moll, P. Arnold, M. Malzer, V. Krozer, D. Pozdniakov, R. Salman, et al., "Radar-based structural health monitoring of wind turbine blades: The case of damage detection," Structural Health Monitoring-an International Journal, vol. 17, pp. 815-822, Jul 2018. 
[5] T. Y. Hsu, S. Y. Shiao, and W. I. Liao, "Damage detection of rotating wind turbine blades using local flexibility method and long-gauge fiber Bragg grating sensors," Measurement Science and Technology, vol. 29, Jan 2018.

[6] M. Mieloszyk and W. Ostachowicz, "An application of Structural Health Monitoring system based on FBG sensors to offshore wind turbine support structure model," Marine Structures, vol. 51, pp. 65-86, Jan 2017.

[7] L. Dolinski, M. Krawczuk, and A. Zak, "Detection of Delamination in Laminate Wind Turbine Blades Using One-Dimensional Wavelet Analysis of Modal Responses," Shock and Vibration, 2018.

[8] R. Marks, C. Gillam, A. Clarke, J. Armstrong, and R. Pullin, "Damage detection in a composite wind turbine blade using 3D scanning laser vibrometry," Proceedings of the Institution of Mechanical Engineers Part C-Journal of Mechanical Engineering Science, vol. 231, pp. 3024-3041, Aug 2017.

[9] Z. Bo, Z. Yanan, and C. Changzheng, "Acoustic emission detection of fatigue cracks in wind turbine blades based on blind deconvolution separation," Fatigue \& Fracture of Engineering Materials \& Structures, vol. 40, pp. 959-970, Jun 2017.

[10] J. L. Tang, S. Soua, C. Mares, and T. H. Gan, "An experimental study of acoustic emission methodology for in service condition monitoring of wind turbine blades," Renewable Energy, vol. 99, pp. 170-179, Dec 2016.

[11] M. Doroshtnasir, T. Worzewski, R. Krankenhagen, and M. Rollig, "On-site inspection of potential defects in wind turbine rotor blades with thermography," Wind Energy, vol. 19, pp. 1407-1422, Aug 2016.

[12] C. Galleguillos, A. Zorrilla, A. Jimenez, L. Diaz, A. L. Montiano, M. Barroso, et al., "Thermographic non-destructive inspection of wind turbine blades using unmanned aerial systems," Plastics Rubber and Composites, vol. 44, pp. 98-103, Apr 2015.

[13] E. Jasiuniene, R. Raisutis, R. Sliteris, A. Voleisis, A. Vladisauskas, D. Mitchard, et al., "NDT of wind turbine blades using adapted ultrasonic and radiographic techniques," Insight, vol. 51, pp. 477-483, Sep 2009.

[14] A. T. F. García Márquez, J. Pinar Pérez and M. Papaelias, "Condition monitoring of wind turbines: Techniques and methods," Renewable Energy vol. 46, pp. 169-178, 2012.

[15] F. P. G. Marquez, A. M. Tobias, J. M. P. Perez, and M. Papaelias, "Condition monitoring of wind turbines: Techniques and methods," Renewable Energy, vol. 46, pp. 169-178, Oct 2012.
[16] Y. Xia and H. Hao, "Statistical damage identification of structures with frequency changes," Journal of Sound and Vibration, vol. 263, pp. 853-870, Jun 122003.

[17] A. May, D. McMillan, and S. Thons, "Economic analysis of condition monitoring systems for offshore wind turbine sub-systems," Iet Renewable Power Generation, vol. 9, pp. 900907, Nov 2015.

[18] Y. W. Ou, E. N. Chatzi, V. K. Dertimanis, and M. D. Spiridonakos, "Vibration-based experimental damage detection of a small-scale wind turbine blade," Structural Health Monitoring-an International Journal, vol. 16, pp. 79-96, Jan 2017.

[19] Z. Y. Shi, S. S. Law, and L. M. Zhang, "Damage localization by directly using incomplete mode shapes," Journal of Engineering Mechanics-Asce, vol. 126, pp. 656-660, Jun 2000

[20] E. P. Carden and P. Fanning, "Vibration based condition monitoring: A review," Structural Health Monitoring, vol. 3, pp. 355-377, Dec 2004.

[21] W. K. Gawronski, Advanced structural dynamics and active control of structures. New York: Springer-Verlag, 2004.

[22] A. Fazlizan, W. T. Chong, S. Y. Yip, S. C. Poh, and W. K. Muzammil, "Double Multiple Stream Tube Analysis of Non-Uniform Wind Stream of Exhaust Air Energy Recovery Turbine Generator," International Journal of Precision Engineering and Manufacturing-Green Technology, vol. 4, pp. 401-407, Oct 2017.

[23] D. Adams, J. White, M. Rumsey, and C. Farrar, "Structural health monitoring of wind turbines: method and application to a HAWT," Wind Energy, vol. 14, pp. 603-623, May 2011.

[24] F. Kanyako and I. Janajreh, Investigating Blade Performance of Small Horizontal Axis Wind Turbine based on Blade Element Momentum Theory, Applications of Information Technology to Renewable Energy Processes and Systems (ITDREPS), 1st International Conference \& Exhibition on the Applications of Information Technology to Renewable Energy Processes and Systems, Morocco, 2013

[25] É. HÉBERT, CÔTÉ, Jean, SMITH, Marc, "A geostatistical analysis of variations of permeability within a compacted dam core," Proceedings of the 17th Nordic Geotechnical Meeting Challenges in Nordic Geotechnic, 25th 28th of May 2016. 